\title{
Engager l'anthropologie du développement à prendre en considération le malaise post colonial
}

Pierre-Joseph Laurent

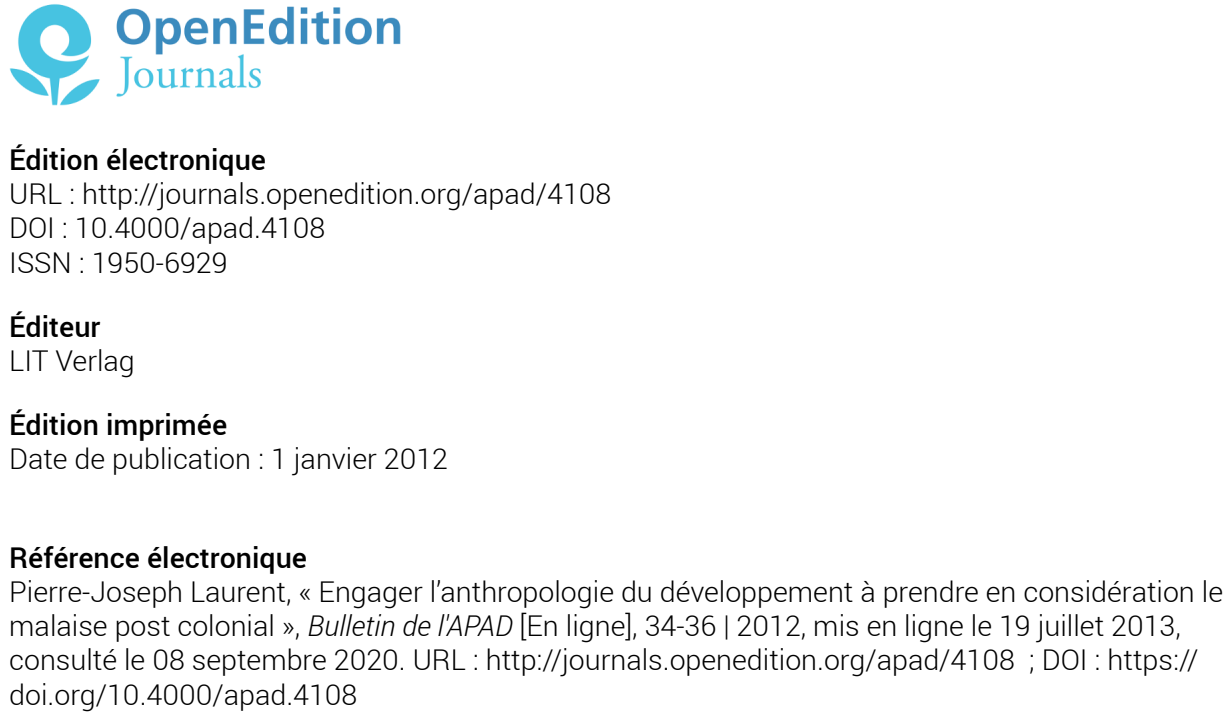

Édition électronique

URL : http://journals.openedition.org/apad/4108

DOI : $10.4000 /$ apad.4108

ISSN : 1950-6929

Éditeur

LIT Verlag

Édition imprimée

Date de publication : 1 janvier 2012

Référence électronique

Pierre-Joseph Laurent, « Engager l'anthropologie du développement à prendre en considération le malaise post colonial », Bulletin de l'APAD [En ligne], 34-36 | 2012, mis en ligne le 19 juillet 2013, consulté le 08 septembre 2020. URL : http://journals.openedition.org/apad/4108; DOI : https:// doi.org/10.4000/apad.4108

Ce document a été généré automatiquement le 8 septembre 2020.

Bulletin de l'APAD 


\title{
Engager l'anthropologie du développement à prendre en considération le malaise post colonial
}

\author{
Pierre-Joseph Laurent
}

\section{Introduction}

1 L'engagement pour de nombreux chercheurs demeure une attitude suspecte dès lors que la démarche scientifique entend se mettre hors de cause pour atteindre l'objectivité et rendre crédible ses résultats. Le point de vue objectif doit s'instituer à l'écart de l'expérience humaine partagée qui est une manière de définir l'engagement. Si l'objectivation s'oppose à ce type d'engagement humain, elle conduit toutefois à un autre engagement, celui de la prise de parole de la science qui - sans véritable concurrence dès lors qu'elle est qualifiée d'universelle et d'objective - occupe une place sur la scène publique. Dans ce type d'engagement, il est plutôt question d'une forme particulière de pouvoir, celui détenu par certains scientifiques lorsqu'on leur demande de discerner le vrai du faux, là où les faits établis doivent finalement faciliter, pour ne pas dire parfois entériner, les décisions politiques lorsqu'elles portent sur le choix de normes et de règles en matière de gestion des sociétés humaines et au-delà des asymétries sociales (l'ordre hiérarchique) rendues acceptables.

2 Si ces spécialistes sont engagés, s'agit-il alors du même engagement lorsqu'on évoque l'anthropologie et son champ singulier que constitue l'anthropologie du développement et du changement social ? Mais alors, comment faire science et être engagé en anthropologie du développement? La question semble anodine, mais l'histoire particulière de la discipline qui a partie liée avec la colonisation, l'évangélisation, l'occidentalisation, voire ensuite avec la promotion de la culture 
des projets promus par les coopérations au développement, fait qu'elle est, d'une manière ou d'une autre, impliquée dans la reproduction des rapports inégaux entre les sociétés, dont ceux particuliers qui instituent les relations Nord-Sud. La question de l'engagement en anthropologie ouvre ainsi la porte à des débats délicats. Ils portent sur les fondements épistémologiques de la discipline autant que sur sa légitimité dans l'espace des sciences.

3 La simple évocation de la notion d'engagement ne peut pas fonctionner comme un talisman qui exonèrerait la discipline de l'autocritique. ${ }^{1} \mathrm{Je}$ me souviens de cet échange avec une femme politique de premier plan d'un pays d'Afrique de l'Ouest laquelle agacée par les conseils d'un consultant militant européen se demandait, l'air triste, mais décidé, d'où il tirait sa légitimé pour lui rappeler ainsi ce que signifie être engagée ! Sans une analyse approfondie et critique de l'engagement en anthropologie ${ }^{2}$, il est illusoire de pouvoir articuler les deux domaines qui en sont pourtant la condition, c'est-à-dire la connaissance scientifique et la nécessité pour ce faire d'une expérience humaine engagée.

4 L'anthropologie est une science engagée lorsque, revendiquant sa singularité face à l'universalité, elle expose sa finalité. En effet, l'engagement de l'anthropologie repose sur l'expérience humaine, comme la condition pour faire science, dès lors que le terrain est l'expérience de l'autre, là où la reconnaissance de l'altérité (au moins une fois dans son existence de chercheur) devient la condition heuristique pour instruire la démarche comparative. Celle-ci s'ancre d'abord sur le respect du sens accordé aux savoirs transmis par les personnes rencontrées sur le terrain, durant un moment particulier.

5 Dans ce texte, je voudrais présenter des jalons pour une anthropologie résolument préoccupée par des enjeux épistémologiques et éthiques renouvelés au contact des terrains du XXIe siècle lesquels posent de lourds défis à la discipline dont ceux portés par la critique postcoloniale ${ }^{3}$ et la nécessité de s'émanciper du terrain conçu comme une entité close afin de sortir définitivement de l'exotisme. ${ }^{4 .}$ L'impossible engagement de l'anthropologie sans la prise en considération d'un double contentieux. Impliqué depuis la création de l'APAD dans des recherches sur le développement et le changement social, ayant, avant ma carrière universitaire, piloté des projets de développement et accepté des missions d'expertise comme agronome et anthropologue, c'est en connaissance de cause que je voudrais balayer devant ma porte.

\section{Expérience d'anthropologue}

6 C'est donc assez lentement que je suis parvenu à abandonner l'idée de faire le développement, c'est-à-dire d'avoir des projets pour autrui, condition pourtant sine qua non pour (re)connaître l'autre et songer à une carrière d'anthropologue. Au Burkina Faso, mon expérience de terrain avec les habitants de Kulkinka (un village situé dans la province de l'Oubritenga sur le plateau central mossi) fut aussi celle d'un engagement (passionné et passionnant) auprès d'une association paysanne qui en était à ses balbutiements. J'ai vécu ce premier terrain anthropologique comme une expérience humaine forte et bouleversante. Elle équivaut à une conversion « allant vers ", formule qui exprime ma transition du métier de développeur à celui 
de chercheur, ainsi que ma découverte de l'altérité. Celle-ci renvoie à ma prise de conscience, avec toutes les conséquences que cela implique, de l'existence de réalités autres, incompressibles. Cette altérité m'était quotidiennement rappelée dès lors que j'étais incapable (et encore aujourd'hui) d'anticipations, c'est-à-dire de comprendre a priori les raisons de certaines actions des villageois sans autres explications de leur part. Cela se caractérisait parfois par des débats houleux qui pouvaient donner lieu à de véritables palabres qui exprimaient sans doute ma lassitude de ne pas pouvoir accéder à leur monde. À elle seule, cette difficile anticipation justifie déjà pleinement un terrain de longue durée. À l'issue de l'expérience, je me suis pourtant senti comme un autre. Je n'étais plus vraiment là où je devrais être, car mes anciens commanditaires, ne me reconnaissant plus, m'ont écarté. Consécutivement à cette éjection du monde des interventions en développement, je suis rentré à l'académie. Dans l'intervalle, j'avais transité du rôle de conseiller, c'est-à-dire de celui qui sait, généralement sourd à l'autre en raison de certitudes, à une posture qui allait progressivement me pousser à rester derrière les villageois, c'est-à-dire à apprendre leurs gestes et à écouter leurs paroles. C'est sur la pointe des pieds que je participais et parvenais parfois à apercevoir, pour paraphraser Clifford Geertz, ce qui faisait sens pour eux. ${ }^{5}$

7 Ainsi dès mon arrivée sur ce terrain en 1988, pour mon premier long séjour (de trois ans et demi), dans le village de Kulkinka ${ }^{6}$, je fus victime d'un empoisonnement. Je m'étais installé dans la concession d'un chef de segment de lignage (Zoundou Zoungrana du quartier de Bissiga). Resté fidèle aux cultes coutumiers, il parlait français et nous avions sympathisé. J'ignorais à ce moment qu'il avait un grand-frère influant, de surcroît pasteur pentecôtiste. En tentant de m'empoisonner, les villageois, majoritairement musulmans, m'indiquaient, à leurs manières, que si je voulais m'établir à Kulkinka, je devrais m'intéresser à tous les quartiers et pas à une famille en particulier. D'emblée, je me trouvais impliqué dans les polémiques qui animaient les relations entre les quartiers du village. Je serai évacué par quelques jeunes du village vers un petit centre urbain. Plus tard, ceux-ci m'exhorteront de revenir, m'expliquant, pour me convaincre, qu'un devin (kinkirbaaga) avait identifié le coupable et qu'un féticheur (tiim mor soaba) l'avait paralysé. L'attaque avait été ainsi vengée.

8 Je me souviens encore qu'à la même époque se structurait dans ce village, la fédération Wend-Yam. Elle deviendra rapidement une association importante du pays. Les membres fondateurs se réunissaient de nuit et en secret. Les rassemblements étaient interdits dans ce moment postrévolutionnaire qualifié de "rectification ». Toutefois les paysans des environs se rencontraient pour débattre de budgets et de cotisations. Lors d'une assemblée générale dont j'ignorais l'existence, les villageois allaient m'élire trésorier adjoint de l'association avec charge de veiller sur les fonds et d'initier leur comptable à une gestion financière. Sans y réfléchir vraiment, j'acceptais ce rôle délicat au cœur des affaires locales. Ce rôle allait m'introduire de manière privilégiée à la société rurale mossi... ${ }^{7}$

9 Cette expérience de terrain, vécue avec les villageois de Kulkinka, fut celle d'un engagement sans réserve dans une association paysanne. S'engager sur le terrain, dans une longue durée, m'a progressivement permis d'épurer mes recherches en fonction de ce qui fait sens pour eux, je veux dire leurs soucis. On le comprend déjà, l'engagement s'associe à l'expérience d'un contexte singulier. Cette expérience 
humaine, c'est-à-dire l'engagement de l'anthropologue, ou encore, la part subjective de cette posture épistémologique est le propre de l'observation participante dès lors qu'elle est la condition pour respecter, au mieux, les données et pouvoir les interpréter. L'interprétation est une inscription, disait Paul Ricoeur, ou encore, une fabrication pour suivre l'épistémologie de Mike Singleton dès lors qu'il est question de reconnaitre son expérience, non tant pour elle-même, mais pour expliquer "là où on veut en venir " (et donc s'engager) ${ }^{8}$. L'engagement en anthropologie est celui de l'aveu de subjectivité ou de singularité : le fait de ne pas pouvoir être hors de cause devient la condition préalable pour dire le vécu des gens, là où le modèle objectivant (c'est-à-dire la simplification ad hoc) laisse croire aux sciences hypothético-déductives qu'elles sont (totalement) hors de cause, c'està-dire désengagée. ${ }^{9}$

10 Dans les points suivants, il sera question d'expliciter la proposition selon laquelle l'engagement en anthropologie est d'abord celui du risque de l'interprétation ${ }^{10}$. Cela conduit à oser dire le sens, c'est-à-dire le sens d'une expérience singulière avec un groupe de personnes. Il apparaît alors que cette forme d'engagement à la subjectivité est la condition pour l'anthropologie de faire science. Toutefois, au risque de ruiner cette expérience, une condition est toutefois préalable ; celle de lever un double contentieux hérité de l'histoire de la discipline et de son implication dans des relations inégalitaires.

\section{Enonciation d'un double contentieux comme préalable à l'engagement}

11 Le premier contentieux est de nature épistémologique. S'engager à avouer ses intentions, c'est-à-dire à expliciter le sens à partir duquel on traite l'expérience du terrain, renvoie à identifier l'ornière que représente l'universalité pour le savoir anthropologique. Il est ici plus particulièrement question du domaine de l'anthropologie lié à l'expérience de terrain laquelle aboutit à la monographie. Avec la monographie, il ne peut être question de catégories hors culture, transculturelles et de nature universelle, car elles biaiseraient le sens du terrain. La question se pose certes autrement en anthropologie fondamentale ${ }^{11}$ ou (peut-être) avec le structuralisme de Claude Lévi-Strauss, supposé être humaniste dans son essence. Bref, au risque d'alimenter le malaise postcolonial, l'autocritique de l'engagement de l'anthropologue demande de soumettre cet engagement à une déconstruction afin de se réengager autrement, c'est-à-dire méthodologiquement en toute connaissance de cause de sa propre singularité de chercheur.

12 D'une certaine manière, le champ de l'anthropologie du développement et du changement social - même si j'en conviens que l'APAD a très largement contribué à l'en extraire ${ }^{12}$ - flirte avec l'aide au développement, non dénuée d'une reproduction des relations inégales entre le Nord et le Sud. Le problème n'est pas celui de l'accession quasi généralisée des peuples à une forme de la globalisation. Il s'agit plutôt de s'intéresser au désir généralisé de globalisation. On pourrait considérer l'idée d'une ascension sociale et qui tendrait vers un désir de consommation sans pour autant que cela soit une conversion totale à l'Occident ? ${ }^{13}$ Autrement dit, l'anthropologie pour faire science dans le domaine du 
développement et du changement social doit se désengager de l'idéologie de l'aide (pour mieux l'analyser), au risque de rester un des fers de lance de l'occidentalisation et laisser ainsi le flanc à la critique.

13 Les critiques formulées par des intellectuels, issus d'univers qui furent colonisés, sont pertinentes. Elles ne se résument pas à vouloir faire payer leur dette aux intellectuels occidentaux en raison de l'implication de leur discipline dans l'histoire coloniale, ni à espérer faire science entre soi, par le simple fait de nier le droit de parole aux allochtones. Certes, des dérives existent, mais ces critiques sont fondamentales. Elles portent sur l'examen des postulats de la pensée postcoloniale dont s'est nourrie l'anthropologie (lorsqu'elle ne prend pas la peine de déconstruire les catégories héritées de la colonisation, voire de l'évangélisation et de certains aspects de l'aide au développement). Dans ces critiques postcoloniales, il est essentiellement question d'un retour sur les postulats épistémologiques de la discipline et plus particulièrement sur son référentiel universaliste au contenu idéologique. L'anthropologie en tant que science n'a pas la vocation de convertir l'autre. L'autocritique de ce type d'engagement constitue l'étape préalable pour esquisser les contours d'une épistémologie adaptée à la discipline. Cela implique l'idée d'un engagement qui fait renaitre la notion de terrain pour traiter d'une expérience humaine, celle de l'altérité.

14 Le second contentieux porte plus particulièrement sur une analyse critique du contexte contemporain dans lequel prend forme l'expérience sur le terrain. Il convient de s'interroger sur la possibilité pratique de l'engagement. Pour ce faire, il est nécessaire de prendre des distances avec la bonne conscience accordée par la militance qui impliquerait qu'à partir d'un lieu particulier, il serait possible de séparer les bonnes des mauvaises causes. Toutes les sociétés pour se reproduire instituent des asymétries, elles sont souvent acceptées et/ou rendues telles.

15 Il sera à présent question de rendre compte en détail de ces deux contentieux.

\section{Autocritique méthodologique de l'engagement comme condition de la connaissance anthropologique}

Beaucoup d'anthropologues ont dû prendre conscience des conséquences de leurs désirs d'aider ceux qu'ils croisent sur leurs terrains. «La foi d'anthropologue » d'un Mike Singleton, par exemple, fut celle qui l'a poussé à élaborer une épistémologie pour déconstruire le démon de la pensée missionnaire là où pour d'autres, ce démon équivalait à aider les pauvres par des projets ${ }^{14}$. Depuis longtemps déjà, par sa critique des formes les plus diverses adoptées par la " pensée missionnaire », le nominalisme anthropologique développé par $\mathrm{M}$. Singleton doit se comprendre comme une réponse à la pensée coloniale et aux études postcoloniales. Elle débouche sur une critique $d u$ savoir anthropologique qu'il qualifie de " nominalisme tempéré $»^{15}$. Cette critique est aujourd'hui saluée par la profession comme une contribution importante au renouvellement du débat en anthropologie. ${ }^{16}$ À l'issue d'un long périple géographique et intellectuel (alimenté par son érudition et ses compétences linguistiques), passant de la théologie à l'anthropologie, Singleton découvrira finalement que le culte des ancêtres n'en est pas un, mais une manière de symboliser la séniorité. De manière similaire, dans son 
sillage, en transitant de l'agronomie à l'anthropologie, j'ai compris qu'à Kulkinka je n'étais pas en présence de partenaires de projets de la coopération au développement, mais de villageois rusant avec des offres de coopération pour arriver à leurs propres fins, par la trahison de l'esprit d'universalité qu'établit l'idée d'une "co-opération». Je propose à présent d'exposer schématiquement l'épistémologie de Mike Singleton.

\section{S'engager dans l'expérience de l'altérité}

17 On n'y échappe pas, l'anthropologie est la science d'un lieu. Elle n'est pas universelle, il n'y a pas de méta-anthropologie, mais une ethno-anthropologie à prétention universelle, c'est-à-dire mue par une volonté de convaincre les autres chercheurs qu'elle est la seule manière légitime de penser scientifiquement l'autre. La critique de Singleton est radicale dès lors que la vérité d'une culture n'est pas crédible. Au contraire, l'anthropologie et l'épistémologie qui la sous-tend résultent d'une histoire, mais d'une histoire qui peut être dépassée. La pratique de l'anthropologie demande de conceptualiser notre propre épistémologie (la situer dans la durée). Il convient pour cela de s'engager, c'est-à-dire d'accepter de s'engager à se retrouver totalement ailleurs et autrement.

18 Pour Singleton, le processus de connaissance en anthropologie passe par l'engagement à oser interpréter son terrain. Sachant que l'interprétation résulte de l'engagement à " reconnaître là où on veut en venir » afin d'expliciter le sens qui nous anime et avouer ce qui semble à nos yeux bon ou mauvais. À l'inverse, penser l'universel renvoi à une convention qui consiste à assimiler l'autre à soi. De cet antagonisme, se noue le premier contentieux exposé ci-dessus. En guise de réponse, Singleton développe une épistémologie sans équivoque: hormis une autocritique de l'engagement en anthropologie, il est impossible de faire science.

19 Le terrain en anthropologie n'est pas une vaine coquetterie, mais une épistémologie. Le terrain comme méthode institue une épistémologie radicalement autre que celle promue par la sociologie, par exemple, dès lors que par l'expérience, le terrain permet de retrouver chez l'autre non pas d'emblée du même, mais de la différence. Rendre ainsi pensable la comparaison, comme le support de la démarche scientifique, n'a rien à voir avec une quelconque dérive culturaliste, mais bien avec une critique de l'universalité d'une culture particulière qui rend impossible le savoir anthropologique, en raison de l'impensable singularité.

20 L'expérience du terrain est celle d'avoir un jour décidé de "lâcher prise». L'abandon même temporaire de ces certitudes, ou encore l'expérience du doute se transforme en une expérience de l'altérité laquelle conduit à se laisser progressivement envahir du sens particulier d'un groupe de personnes comme de la manière de connaître en anthropologie ${ }^{17}$.

\section{Des données et des faits}

21 Le terrain est une étude de cas et chaque cas une étude à part entière alors la théorie devient le plus souvent une généralisation sans grande conséquence sur la 
connaissance générale de la discipline. Le savoir anthropologique (celui consigné surtout dans les monographies, à la différence du domaine particulier que constitue l'anthropologie fondamentale qui a partiellement partie liée avec l'univers hypothético-déductif) relève surtout de la qualité et de l'aspect novateur des faits relatés.

22 Singleton propose de reprendre la distinction fondamentale entre les données et les faits lorsqu'il enseigne que s'il n'y a bien un phénomène, on rencontre de multiples interprétations. De ceci, il tire que l'engagement en anthropologie consiste à oser analyser les tenants et aboutissants de sa propre interprétation comme de la manière de faire science. Les données sont les data et l'interprétation leur actualisation. Sachant qu'un même phénomène donne lieu à différentes interprétations, le réel (les données) n'est pas là, tout prêt à être compris. Le réel n'a rien à dire sans être réalisé. C'est donc bien le point de vue qui fait naître ce qui est vu. Dans ce sens, les choses sont en devenir. L'engagement de l'anthropologie sur son terrain est l'expérience scientifique qui lui permet de «(re)naître avec » et de produire un savoir valide. Les données, c'est-à-dire ce qui est donné (le don, le cadeau) ou ce qui est vu, sont les phénomènes, c'est-à-dire le réel de référence, les choses, les objets. Il est ainsi question de recevoir du dehors (du dehors de soi) la réalité des données. Mais si ces dernières sont les mêmes pour tous - les données sont indiscutables-, les faits eux diffèrent.

23 Les faits renvoient à l'idée de "faire avec" pour faire advenir les choses (facere), soit encore pour réaliser le réel. Dans ce sens, les choses sont en devenir lorsqu'il s'agit de fabriquer, de réaliser le réel. C'est le vécu qui fait naitre la réalité (avant qu'une espèce animale ne soit découverte et identifiée, elle est considérée comme inconnue par la science, pourtant elle existe). Par le terrain qui est une expérience, le réel prend sens dans "la réalité » qui est celle de tout un chacun, c'est-à-dire celle autorisée par un corps et une culture située dans un temps et un espace particulier. Autrement dit, on est toujours en train d'établir ses idées à partir des données.

24 On voit certes les mêmes choses, mais entre deux personnes qui voient ces choses, il existe un "pont ontologique ", c'est-à-dire de l'expérience humaine irréductible, celle d'être au monde et de pouvoir le signifier à partir d'un corps et d'une culture incorporée, située dans une socio-historique. Les données sont perçues, réalisées, conçues, ressenties à la faveur d'un corps propre, comme de la seule manière d'être au monde. ${ }^{18}$ Convenons alors que les données ne deviennent des faits que si elles sont assignées à un lieu de vie (un espace de vie en commun, la culture d'un groupe particulier) par celui que les reconnaît et donc se faisant, les interprète. L'objet est, en quelque sorte, gisant tant qu'aucun point de vue n'est porté sur lui. On produit ce qui est vu. Ce processus exprime au mieux ce que connaître signifie, c'est-à-dire « naître avec » ou « co-nnaître ».

25 De manière concrète, connaître demande de se laisser gagner par la réflexivité lorsqu'il devient indispensable, pour mettre en œuvre l'épistémologie ad hoc de l'anthropologie, qui ne peut se payer le luxe de s'abriter derrière la mathésis, d'établir pour soi, ce que savoir veut dire. Le savoir anthropologique pour s'instituer a dû se rapprocher de l'indicible, lequel renvoie à l'expérience d'une existence humaine partagée qui n'est autre que l'observation participante. ${ }^{19}$ Cette part d'indicible, d'une expérience à devoir décrire, comme l'objet même de la 
monographie, équivaut - pour reprendre un exemple favori de Mike Singleton - à parler de la sexualité ou de vivre une nuit d'amour !

26 Pour le nominaliste, l'existant revient à "faire naître un monde ", ce qui est bien l'intention de toutes monographies. Dans celles-ci, les faits relatés résultent d'un jugement, c'est-à-dire d'un engagement acquis de l'expérience d'un terrain. Pour la pensée engagée, le réel est toujours une réalisation et il ne peut en conséquence y avoir de catégories établies a priori, c'est-à-dire de catégories conçues comme des essences désincarnées, universelles et transculturelles. ${ }^{20}$

27 Comme épistémologie, le terrain colle à la posture scientifique de l'anthropologie. Il est l'expérience humaine qui permet au chercheur d'énoncer le bon du mauvais, en accord avec l'intention de respecter au mieux les données. Pour ce faire, l'anthropologue doit mobiliser une capacité réflexive, engagée dans l'aveu des préalables présidant à l'élaboration de sa propre interprétation. Autrement dit, les faits relatés dans les monographies sont tributaires d'une situation sociohistorique qui résulte d'un jugement établi en référence à une culture donnée. En conséquence, pour prétendre à un niveau de scientificité indiscutable, l'anthropologie doit décrire et comparer les phénomènes en toute connaissance de cause.

28 L'engagement en science n'est plus ici considéré comme suspect à l'inverse de l'autre posture où il s'agissait de mettre l'engagement (la subjectivité) hors de cause (par le recours à la généralisation que permet le modèle) pour atteindre l'objectivité et prétendre à la crédibilité. À la différence de ce point de vue objectif qui s'institue hors du monde (parfois en toute innocence de causes), c'est-à-dire à l'écart de l'expérience humaine partagée, la validation scientifique en anthropologie passe par celle d'un engagement à dire sa subjectivité pour établir plus objectivement les faits dans la vérité d'autrui. L'anthropologie est engagée au risque de commettre une faute méthodologique. Cette faute consisterait à recourir à des catégories considérées comme universelles alors qu'elles expriment seulement la manière de signifier les choses à partir d'un lieu particulier. Autrement dit, les choses sont des causes crédibles parce qu'elles sont conventionnées par une culture. L'expérience du terrain, sous-tendue par la "rigueur du qualitatif» pour reprendre la formule de Jean-Pierre Olivier de Sardan $^{21}$, est la méthode qui permet de respecter le sens des données parce que cette expérience les enracine dans le vécu des gens. L'engagement pour l'anthropologie consiste à apprendre à déconstruire ses catégories afin de reconnaitre un autre sens, avec l'objectif de le relater au plus juste.

29 L'engagement implique que l'anthropologie ne peut pas être tenue hors de cause. Le chercheur sur le terrain est incarné dans son corps qui est son outil pour effectuer le monde qui l'environne et le reconnaître. En suivant Mike Singleton, il est moins question d'embrasser l'universel par des généralisations que d'oser s'engager à décrire un cas particulier en avouant - comme la garantie de ce qui se fait de mieux en matière d'interprétation - sur quoi porte le jugement en guise de protocole scientifique qui conduit à la validation, au contrôle et facilite la reproductibilité.

30 Engager l'anthropologie revient à reconsidérer les raisons implicites situées à ses fondements épistémologiques, afin de reconnaître que la discipline n'est pas 
hors de cause d'une pensée postcoloniale. Finalement, Singleton démontre que l'anthropologie dispose déjà, si elle y met la forme adéquate, peut-être plus que d'autres disciplines, d'une épistémologie capable de la prémunir de l'idéologie qui assigne les faits à une culture donnée.

\section{Engagement éthique dans la société à big men de la modernité insécurisée}

31 Je viens de montrer que ce premier contentieux porte sur l'engagement de l'anthropologue comme d'une obligation méthodologique indispensable au respect des données et de la production de faits crédibles. Pour faire science, l'engagement permet de considérer que l'anthropologie est d'abord la science d'un lieu. La critique épistémologique de l'universalisme - dans le sens ici restreint de la pensée hégémonie - accepte la critique postcoloniale par un retour critique sur les fondements épistémologiques de la discipline, non pas en générale, mais en pratique $^{22}$. Chaque chercheur est concerné lorsqu'il est invité à débusquer la manière dont les postulats de la pensée postcoloniale s'enracinent dans tout un chacun. Autrement dit, avec l'épistémologie de Mike Singleton ce n'est pas l'anthropologie en général qui est engagée, mais chaque anthropologue, au risque, s'il ne le fait pas, en guise de production scientifique, de ne reproduire dans ses interprétations que la trame des catégories d'un lieu particulier, c'est-à-dire le sien, passant par là à côté de l'objectif de la discipline qui consiste dans la connaissance de l'autre. L'anthropologie en tant que discipline scientifique n'est pas engagée, mais chaque anthropologue doit l'être pour être reconnu comme tel. Ceci étend rappelé, il sera à présent question de discuter d'un second contentieux celui de la nature d'un engagement concret auprès d'acteurs sur le terrain. Intimement relié au premier, ce second contentieux porte sur le choix d'un engagement éthique de l'anthropologue sur le terrain.

32 À partir de l'exposé d'une étude de cas, la discussion du second contentieux traite d'une analyse critique du contexte contemporain dans lequel prend forme l'expérience sur le terrain. Il sera question de s'interroger sur les conséquences pratiques de l'engagement. Pour ce faire, en relation avec ce qui vient d'être dit, il ne peut être question de se satisfaire d'une forme de militance capable de juger a priori les bonnes des mauvaises causes (cf. par exemple, à partir d'un engagement vécu comme une évidence internationalement partagée et in-questionnée puisque garantie par la bannière de la "critique de gauche»), car elle ne prend pas en considération le malaise postcolonial, ni généralement la capacité d'agir des groupes de personnes avec qui l'anthropologie partage son expérience de terrain ${ }^{23}$. J'ai déjà indiqué que la simple évocation de la notion d'engagement ne peut pas fonctionner comme un talisman qui exonèrerait de l'autocritique, l'engagement de l'anthropologue sur le terrain. Dans la mesure où s'engager et résister adoptent des formes variables et souvent insoupçonnées, les « opposants » ne sont pas toujours ceux auxquels on pense, car toutes sociétés pour se reproduire instituent ses propres asymétries, souvent acceptées et/ou rendues comme telles par les groupes dominants. Il est donc délicat, pour l'anthropologue sur le terrain, de s'engager en référence à son univers - par exemple celui régi en termes de bien et de mal, selon 
des normes considérées comme transculturelles, mais promues à partir d'un lieu particulier.

33 De mon expérience sur des terrains dans certaines régions d'Afrique, dans un contexte que j'ai qualifié de modernité insécurisée (cf. ci-dessous) ${ }^{24}$, où règnent en maître les réseaux le clientélisme et la dépendance, j'ai rencontré quelques bigmen, mais surtout de nombreuses autres personnes sans grade. Dans ces « sociétés à big-men ", pour les nommer ainsi, caractéristiques d'un moment particulier du développement de certaines régions d'Afrique, avant de pouvoir s'engager, il s'avère important d'en comprendre les grands principes de fonctionnement. Dans des sociétés où les politiques publiques sont parfois inexistantes pour des pans entiers de la population qui peut se permettre de ne pas s'impliquer dans ce mode "de vivre ensemble ", au risque de s'exclure de la socialité ou tout simplement de la survie? L'anthropologue peut raconter l'univers des big-men, mais avec qui s'engager dès lors qu'il y a beaucoup de perdants qui sont souvent aussi des dépendants, des clients! Ainsi ce cas, d'un vol dans la caisse d'une association villageoise qui m'a été raconté pour me faire comprendre que les choses ne sont pas simples en matière de gestion de l'argent issu de projets de coopération au développement en milieu rural sur le plateau mossi du Burkina Faso.

34 Oumar Compaore (animateur de la Fédération Wend-Yam, Kulkinka, 1999: Après ce vol, les membres du comité de gestion ont décidé de partir chez le grand marabout de Nagréongo. Comme il est très fort, pensaient-ils, lui pourra désigner le coupable. Le marabout a écouté, mais il "n'indexa" personne. Il expliqua que les membres du comité connaissaient celui qui avait causé le problème. Ils ont alors compris que le secrétaire était coupable. Le marabout a alors conseillé de ne rien tenter contre lui, car ils (les villageois) pourraient tuer (eux-mêmes) tout le village. En effet, dans la mesure où le secrétaire a volé de l'argent de la caisse pour acheter des choses, des choses qu'il a sans doute ensuite données à plusieurs autres personnes au village. Dès lors, si un fétiche est dirigé contre lui en particulier, il risquerait de se retourner contre tout le village.

Si ce marabout aide les villageois à désigner ouvertement leur coupable, il tente ensuite de socialiser ce vol, à la faveur d'un retour au consensus, par l'habile suggestion que, même à leur insu, tout le monde pourrait avoir profité des larcins. Rien n'est simple : qui est hors de cause ? Avec qui s'engager? Et pourtant, loin de la logique bien/mal d'un Occident capable de discriminer les engagements légitimes, il y a ici aussi des gagnants et des perdants et des bifurcations à prendre, comme celle d'un choix éthique à l'engagement de l'anthropologue sur le terrain.

36 Pour bien fixer les choses à partir de faits établis du terrain, je relaterai ce second exemple. Je l'avais utilisé pour débattre de la notion de bien public dans le cadre de principes démocratiques importés et souvent imposés, par le fait même du mode de gestion que nécessitent les projets soutenus par les offres de la coopération au développement. Je montrais que ces projets peuvent être parfois dissimulés des pratiques et une capacité d'agir liées à un contexte singulier ${ }^{25}$.

En mai 1998, je fus témoin de l'histoire suivante. 
membres de l'association, du petit matériel aratoire pour la production de légumes. Ce matériel devait être acheté déjà fabriqué auprès d'un grand commerçant de la capitale.

39 Entre temps, A., un petit frère du président de la coopérative, a entendu parler de ce financement. Rapidement, il m'a demandé de l'accompagner chez un importateur de matériaux de construction. Là, nous avons commandé pour 12 millions de CFA de fournitures diverses (tôles, barres de fer...) et d'équipement. Nous voulions fabriquer nous-mêmes ces outils agricoles au lieu de les acheter tout fait auprès d'un commerçant. Avec mon appui, A. avait l'intention de monter son propre atelier de soudure. De retour chez son grand frère avec la facture de 12 millions, A. va l'obliger à payer. Comme il est connu et qu'il ne veut pas prendre le risque de nuire à sa réputation (cf. ci-dessous la figure du big-man), il céda à la pression de A.. À charge à présent de A. de fabriquer les outils. A. créa son atelier et demanda à un soudeur et à deux apprentis embauchés de confectionner les outils dans les quantités prévues par le projet.

40 Il faut comprendre que par rapport au budget initialement prévu par l'ONG finlandaise, ils ont ainsi économisé 6 millions de CFA. A. a alors proposé à son grand frère et à moi de partir au Ghana pour acheter avec «son » bénéfice un tracteur. Actuellement, ce tracteur est celui que tu as vu tourner hier en ville.

Dans la conversation qui suivit, l'informateur m'explique que le tracteur est (presque) devenu la propriété de A. ${ }^{26}$ Et il insistera pour me convaincre que vis-àvis de l'ONG finlandaise, le contrat a été clairement rempli, même lorsque je lui suggère qu'ils ont probablement dû faire établir une facture de 18 millions dès lors qu'ils n'en ont dépensé que 12, afin d'acheter le tracteur avec le bénéfice réalisé. Il m'expliquera qu'il ne fut pas question de ristourner l'économie tirée de la fabrication en régie auprès des membres de la coopérative par des activités décidées en assemblée par exemple, voire de débattre d'une réaffectation du solde avec le bailleur de fonds. Il insista plutôt sur l'idée que ce sont des gens jaloux qui tentent de discréditer le président de la coopérative et le nom de A. Relevons encore que les membres de coopérative ne demanderont pas de comptes à leurs responsables, mais ils évoqueront la jalousie pour expliquer les rancœurs de certains d'entre eux vis-à-vis des pratiques de $\mathrm{A}$.

$42 \mathrm{~A}$ partir de ce récit qui sert d'ancrage au raisonnement, je présenterai quelques éléments de ce contexte afin d'alimenter le débat concernant ce deuxième contentieux, celui d'un engagement éthique de l'anthropologue en mesure tenir compte du malaise postcolonial. L'idée de modernité insécurisée peut nous y aider. Cette notion, qui renvoie à un contexte particulier, rend compte d'un moment de réforme des cultures, où un grand nombre de peuples sont aspirés par la modernité trans-nationalisée. Dans la société rurale mossi du Burkina Faso, là où les principes de la sécurité sociale, économique et culturelle de la société coutumière mossi n'existent plus vraiment, abandonnés à la faveur de l'adhésion massive de la paysannerie à une forme de la globalisation, mais où l'Etat-nation n'est pas, pour autant, en mesure d'assurer l'instauration généralisée d'une forme nouvelle de la sécurité sociale (retraite généralisée, aides publiques, chômage...), y a-t-il, malgré tout, la possibilité d'accumuler pour soi et ses proches sans la protection d'un Étattiers garant du bien-être de ses membres et surtout avec quelles conséquences ? Avec la modernité insécurisée, je fais l'hypothèse d'une réponse positive, à la 
condition toutefois d'accepter l'idée d'une société dont les rapports sociaux sont violents, là où la partialité de l'Etat et de la peur de l'autre sont autant d'éléments qui composeraient le lien de société. Y a-t-il dans ce contexte une place pour l'anthropologue engagé?

\section{La confiance et l'entente comme composantes de la sécurité sociale coutumière}

Pour approcher la modernité insécurisée, il est utile de se rappeler des principaux axes qui régulent (régulaient) le «vivre ensemble» au sein d'une société coutumière, mossi en l'occurrence ici. Partons de la proposition selon laquelle l'échange généralisé impliquait la stabilité du groupe envers lequel chaque personne s'était endettée, c'est-à-dire s'était appauvrie, afin de s'enrichir d'un entourage qui jouait un rôle notoire dans l'établissement de la sécurité économique et sociale. La survie de chacun résultait alors de la confiance (kis-sida ou basêm yam en mooré la langue des Mossi) qu'il pouvait accorder aux autres, surtout aux parents, dans le sens ici de la parenté élargie. Les relations de parenté (à la fois le patrilignage: buudu, les consanguins, et les alliés, rembamba) jouaient un rôle déterminant dans l'instauration de la sécurité des membres. Ces relations pouvaient entrer en conflit avec le désir d'accumulation pour soi. On rencontre donc une situation où la confiance (kis-sida) est essentiellement réservée aux relations de parentée ${ }^{27}$ et l'entente (wuum taaba ${ }^{28}$ ) - qui ne peut pas être confondue à kis-sida, la confiance -, est instaurée entre les différents lignages qui constituent la société. Cette idée de confiance par la parenté est bien traduite par le vocable rog yissintomé, (litt. rechercher des liens dans la parenté).

44 Pour sa part, la notion d'entente (wuum taaba en mooré) réside dans un arrangement entre les lignages pour juguler les inévitables conflits inhérents à la vie collective, elle s'établit, ce qui est notoire, sans pour autant vider la querelle entre les groupes. Cette notion ne porte pas uniquement sur un mode singulier de gouvernement des personnes puisqu'elle participe aussi à la production de biens particuliers. L'entente comme mode de relations entre les lignages qui composent la société vient contrecarrer les forces centripètes propres aux rapports de confiance établis entre parents. Ainsi, l'espace lignager possède une tendance à la rétention des biens qu'il est possible d'assimiler à des biens privés.

45 A côté de ce qu'il conviendrait d'appeler les «biens privés lignagers », les biens collectifs représentent le sentiment que possèdent les membres d'un lignage (il s'agit généralement d'un segment de lignage) que les biens privés de chaque autres lignages résidant dans leur village (par exemple) pourraient aussi leur appartenir. Autrement dit, le principe de la sécurité repose ici dans la production, avant tout imaginaire, d'un accès toujours envisageable dans la pénurie, aux biens des autres lignages. Dans ce sens, l'entente limite l'appropriation, définitive, pleine et entière, des biens par un lignage et instaure un consensus qu'il est dangereux de contester. Relevons qu'il est bien question ici d'un imaginaire de biens collectifs, possédés par une communauté, mais de manière individuelle, dans la mesure où ces biens ne cessent pas pour autant d'appartenir à un segment de lignage particulier. Ce processus définit ce qu'il conviendrait de nommer « l'espace public coutumier ». Et 
si on pousse cette idée jusqu'à son terme, il est possible de montrer que c'est le degré d'accumulation d'une collectivité prise dans son ensemble qui détermine le niveau de sécurité de chacun de ses membres.

46 L'édification de la sécurité sociale et économique entre un groupe de personnes est donc ici partiellement liée à une production (essentiellement) imaginaire de biens collectifs (lesquelles renvoient à la permanence de réseaux sociaux et à l'univers de la dépendance mutuelle). Cette forme de la sécurité sociale et économique repose sur l'entretien permanent d'une forme de consensus qu'incarne l'idéologie de l'entente entre les lignages. Ce qui compte c'est de maintenir active la potentialité de l'accès aux biens d'autrui. Mais bien entendu, cet accès n'est ni automatique ni vertueux et là réside toute la complexité d'un processus qui compose avec la rancœur, la peur et la jalousie. ${ }^{29}$

\section{Le sentiment de défiance propre à la « modernité insécurisée »}

Ce qui se transforme avec l'urbanisation récente et rapide de cette région d'Afrique et l'émergence des villes, c'est la manière de vivre en commun. Lorsqu'on change de lieu pour passer du village à la ville, on change aussi de logique, transitant d'une situation où l'on se connaît personnellement, à celle d'un anonymat qui conduit à une certaine atomisation du social. Le vivre ensemble tient ici dans la relation singulière qu'entretiennent des groupes de population avec l'Etat, relation qui a finalement favorisé l'émergence d'une forme de la modernité que j'ai qualifiée d'insécurisée.

48 Dans ce contexte de modernité insécurisée, où l'Etat est autoritaire (à la fois omniprésent et absent - les politiques sociales sont quasi inexistantes) et la prise de parole sur la scène publique problématique, j'ai proposé l'hypothèse d'une gestion du "vivre ensemble", qui assez logiquement, mobilise des catégories de l'imaginaire. Les registres de la peur et la nécessité de protections contre ceux qui vous en veulent, poussent à recourir à des forces invisibles qui, symbolisent au mieux ici, les relations aux autres, en l'absence d'un Etat tenant effectivement le rôle du tiers impartial. ${ }^{30}$

49 La production d'une société basée sur la défiance repose sur un face à face permanent entre les personnes qui doivent - en l'absence d'institutions étatiques réellement tierces -, pour survivre, mettre en scène, de manière permanente et ostentatoire, leur force (d'agression ou de protection), réelle ou/et imaginaire (magique), comme médiateur de la relation aux autres. Les chemins classiques de l'ascension sociale s'amenuisent (écoles, famille, travail salarié) et poussent de nombreuses personnes vers la débrouille, augmentant ainsi le sentiment général d'insécurité. La crainte, la jalousie, la peur s'amplifient et conduisent les populations à rechercher une autre protection (par l'affiliation à des réseaux) que celle qu'aurait pu fournir aux citoyens l'Etat-nation (par la garantie pour tous de la paix sociale, l'accès généralisé à l'enseignement et au système de santé, voire aux différentes formes de l'aide sociale). Le lien social s'édifie donc partiellement sur la possession personnelle d'entités protectrices (fétiches ou conversion à un Dieu puissant capable de protéger de ceux qui vous en veulent et/ou d'affecter ceux qui 
vous oubliant vous laissent dans le besoin). Cette situation entretient un certain climat de peur où chacun se méfie des autres. Ce moment de défiance renvoie à une société largement vouée à la survie de l'individu. Cette survie repose sur la capacité personnelle à mobiliser des ressources, mais également les puissances protectrices, de même que de se montrer capable d'afficher de manière ostentatoire son accès à la consommation et sa capacité à se protéger des jalousies qu'il suscite ${ }^{31}$. Et de manière positive, j'indiquerais ci-dessous que cette situation assez inédite permet de rendre compte d'une autre manière de "vivre ensemble » dans un contexte de globalisation.

\section{Le big man de la modernité insécurisée}

Un personnage que j'ai qualifié de big man acquiert du pouvoir s'il parvient à accaparer une ressource à l'origine d'une rente ${ }^{32}$. Et le big man s'institue par sa capacité à organiser la gestion de la rente, dont une partie alimente des réseaux. Les différents réseaux fabriquent le big man. Et les réseaux qui l'entourent possèdent en commun la capacité de l'obliger tout en soumettant à lui les personnes qui bénéficient de ses largesses. La rente repose donc sur un processus qui inclut l'individu dans le réseau du big man, en le transformant de celui qui cherche à l'obliger en vue d'un bénéfice à celui qui dépend de lui s'il l'obtient (ce processus est réversible ai-je montré dans les deux exemples présentés ci-dessus). Les réseaux de dépendants sont bien consubstantiels à l'organisation de la rente par le big man. De sorte que les réseaux constituent non seulement le faire-valoir du leader, mais représentent une partie nécessaire d'un tout. Le big man n'est donc que l'aiguilleur de la rente, sujet et otage de celle-ci; il ne serait rien sans les réseaux et vice versa. L'appauvrissement conclut généralement cette logique lorsque les sources de la rente se tarissent. ${ }^{33}$

51 A la lisière des logiques du don/dette/dépendance (une des caractéristiques de la sécurité populaire) et de la corruption, dont la frontière n'est jamais clairement délimitée, se tient donc la figure du big man. Ce personnage ambivalent renvoie au tandem obligation (le big man est en quelque sorte obligé de redistribuer des ressources en fonction des pressions exercées par les membres des réseaux qui l'entourent, l'honorent, l'aident et le plébiscitent) et dépendance (soit des membres des réseaux que le big man rétribue selon des critères totalement inégalitaires dont il tire autorité et pouvoir), ce qui équivaut à l'instauration d'un rapport social inégal, basé sur la soumission.

52 Ces logiques peuvent être décrites en termes de corruption et dans ce cas l'engagement éthique de l'anthropologue s'apparente à une forme de dénonciation de la mauvaise gestion. Cette dénonciation ne peut toutefois se faire qu'en référence au lieu particulier qui a établi les règles de la bonne gouvernance. Mais on peut également voir les choses autrement et plutôt que de s'engager à dénoncer a priori, on peut aussi s'engager à comprendre comment s'organise dans ce contexte la vie en commun, notamment pour les cohortes de sans-grades.

53 Dit autrement, ce contexte est aussi celui d'un entre-deux mondes, où les normes issues des différentes sources de pouvoirs (pouvoirs étatiques, coutumiers, communaux, religieux, mais également d'instances internationales, etc.) 
coexistent, s'empilent, s'entrechoquent, se disqualifient, en même temps qu'apparaissent des troubles, parfois violents, de l'identité pour les personnes. Mais au-delà, il est important de relever que les groupes de populations parviennent à fréquemment dépasser, ou plutôt, à composer avec «l'empilement normatif", producteur d'insécurité (pour les plus faibles) en raison de la compétition entre différents systèmes hiérarchiques, pour forcer malgré tout un type d'accord singulier autour de la nécessité de "vivre ensemble» entre les diverses composantes de la société. ${ }^{34}$ Autrement dit, il n'est pas impensable de parvenir à tirer son épingle du jeu malgré "l'enchevêtrement » des logiques sociales $^{35}$. Loin de la normativité, il est alors question de décrire des mouvements complexes de solidarité, capables d'inventer des formes efficaces de la survie urbaine, par exemple par des pratiques festives, religieuses, faites de solidarités, de dons, de dettes et dépendances, mais aussi d'obligations, de soumissions, de mises en danger (de soi des corps) et de violences.

54 Dans un même ordre d'idée et pour conclure, j'évoquerai cette analyse de l'issue désastreuse d'un projet de coopération au développement dont je proposais pour en comprendre le sens de distinguer le détournement et de la corruption. ${ }^{36} \mathrm{Je}$ proposais de réserver au terme de détournement à l'utilisation par un groupe composé de parents, voisins ou amis - de biens collectifs (ceux d'un projet de coopération au développement en l'occurrence ici) à d'autres fins que celles initialement convenues entre les parties, à la faveur des pressions que ce groupe pouvait exercer à l'encontre d'un responsable d'une association (un big man local dans ce cas) qui ne cessait pour sa part de dépendre de cet entourage. La dimension collective a ici de l'importance, car ces détournements conduisent généralement à une socialisation des ressources selon des arrangements locaux subtils et généralement assez consensuels. Dans ce sens, le détournement est tout autant le fait du big-man que de son entourage qui le maintient sous son emprise. Pour résumer cette situation de double dépendance, j'évoquais l'idée du «dilemme du gestionnaire ». Et à l'opposé, je proposais de réserver la notion de corruption pour désigner une volonté manifeste d'enrichissement personnel basée sur l'utilisation de biens collectifs, c'est-à-dire le cas ici d'une rétention pour soi. En conclusion de l'analyse de ce projet de coopération qui n'a certes pas survécu aux détournements, je montrais que ces villageois tenaient portant ce projet (détourné à d'autres fins que celles initialement prévues) comme la condition de départ d'un certain développement de leur zone. En conclusion de ceci et au regard de mon expérience à Kulkinka, tenu à l'écart des big-men de carrure nationale, mais en proximité avec la fédération Wend-Yam naissante, je me suis engagé à cette époque progressivement en meilleure connaissance - à ne pas trahir leur lieu par une dénonciation par trop véhémente. Prenant fait et cause pour ce que j'avais appelé "le dilemme du gestionnaire villageois ", j'ai alors travaillé à le comprendre afin d'établir les forces et les faiblesses de ce système de gouvernance dans le référentiel qui était le sien.

\section{Conclusion}

55 J'ai indiqué que le point de vue objectif qui institue la science en la situant hors de monde tente de la positionner à l'écart de l'expérience humaine partagée. Restons 
attentifs au fait que l'objectivation pure et simple qui met la science hors de cause s'oppose à une autre objectivation, celle qui, reconnaissant le fait de l'engagement humain, inclut la subjectivité dans la démarche de connaissance. ${ }^{37}$

56 J'ai montré comment le point de vue objectif doit s'instituer à l'écart de l'expérience humaine partagée. Cette objectivité peut aussi avoir comme enjeu la prise de parole légitime. Convenons que cette prise de parole et le type d'engagement qui en découle peuvent parfois émanciper ce scientifique, que l'on peut alors qualifier de politiquement engagé, du cadre strict imposé par la méthode qui reste pourtant ce qu'il met en gage pour garantir la véracité de ses analyses (puisque cette méthode ne prend pas vraiment en considération la part subjective du chercheur). Il est alors question de pouvoir, voire parfois d'idéologie, lorsqu'on pousse la science occidentale à dire le vrai du faux, là où les faits établis servent finalement la gestion politique ${ }^{38}$. Si l'objectivité scientifique s'instaure généralement en éliminant le sujet, à l'inverse, l'anthropologue sur le terrain, à la recherche de la rigueur pour sa discipline, s'engage en raison de principes méthodologiques et éthiques.

57 En effet, sur le terrain que ce soit « ici » ou « là-bas », les anthropologues sont souvent confrontés à des questions de sens, de choix éthique, d'engagement, de déontologie, d'implication qui nécessitent des décisions souvent difficiles à prendre en raison des relations nouées et souvent complexes. Ces choix ont des implications évidentes pour le chercheur et la production scientifique.

58 Par l'énoncé de deux contentieux qui portent sur une critique des postulats de la pensée postcoloniale, j'ai indiqué que l'expérience humaine du terrain est la manière de faire science, dans ce champ du savoir. Ce point de vue, implique une posture scientifique particulière où les anthropologues savent, sans doute mieux que quiconque, qu'il serait vain de se départir de la responsabilité. Il est ainsi question d'appréhender les conséquences de l'aveu de subjectivité (qui consiste à expliquer l'implicite de ses motivations) lequel aveu participe à la posture anthropologique (observation participante, comparaison, interprétation), dans le sens englobant où il implique la responsabilité humaine. Celle-ci renvoie aux relations, aux formes de sympathies et de solidarités qu'a su établir l'anthropologue, dont le métier consiste d'abord en une pratique relationnelle qui est à une expérience engagée comme méthode scientifique..$^{39}$

59 Dans la pratique de sa discipline, l'anthropologue peut-il être quitte de l'autre ou est-il "naturellement" impliqué dans le destin de ceux qu'il tente de connaître afin d'en raconter le sens? L'anthropologie est consubstantielle d'une démarche ouverte vers l'autre. Dès lors l'altérité, la relation et la responsabilité font partie du principe de connaissance mobilisé par la discipline qui inclut à l'évidence l'éthique et le devoir dans son épistémologie. 


\section{BIBLIOGRAPHIE}

Agier, M. 2004. La sagesse de l'ethnologue, Paris, L'œil neuf.

Assayag, J. 2005. La mondialisation vue d'ailleurs. L'Inde désorientée, Paris, Seuil.

Bensa, A. 1995. De la relation ethnographique. A la recherche de la juste distance, Enquête, $\mathrm{n}^{\circ} 1$, 1995, pp. 131-140.

-. 2006. La fin de l'exotisme. Essais d'anthropologie critique, Toulouse, Anacharis.

Bhabha, H. K. 2007. Les lieux de la culture: Une théorie postcoloniale, Paris, Payot.

Bierschenk, T. 1991. Les projets et les politiques de développement sont-ils des préoccupations légitimes de l'anthropologie ?, Bulletin de l'APAD, 1991, n¹,

Chauveau, J.-P. 1992. Le « modèle participatif » de développement rural est-il « alternatif »? Eléments pour une anthropologie de la culture des « développeurs », Bulletin de l'APAD, n³, pp. 20-30.

Blundo, G., Olivier de Sardan, J.-P., 2003. Pratiques de la description, Paris, EHESS, Enquête, n³. Bourdieu, P. 1993. Comprendre, in Bourdieu, P. (sous la dir. de) La misère du monde, Paris, Seuil, pp. 903-939.

Butler, J. 2005. Trouble dans le genre. Le féminisme et la subversion de l'identité, Paris, La découverte. Da Matta, R. 1983. Carnavals, bandits et héros : ambiguïtés de la société brésilienne, Paris, Seuil.

de Certeau, M. 1975. L'écriture de l'histoire, Paris, Gallimard.

Descola, P. 2005. Par delà nature et culture, Paris, Gallimard.

Garcia-Canclini, N. 1995. Culturas hibridas. Estrategias para entrar y salir de la modernidad, Editorial Sudamericana, 2da edición, Buenos Aires.

Geertz, C. 1996. Ici et Là-bas. L'anthropologue comme auteur, Paris, Métaillé.

-. 1983. Bali. Interprétation d'une culture, Paris, Gallimard, (notamment, Jeu d'enfer. Notes sur le combat de coqs balinais), pp. 166-215.

Godelier, M. 2004. Métamorphoses de la parenté, Paris, Fayard.

Gould, S. J. 1989. La vie est belle. Les surprises de l'évolution, Paris, Seuil.

Kilani, M. 1994. L'invention de l'autre. Essais sur le discours anthropologique, Lausanne, Payot.

Lahire, B. 1996. Risquer l'interprétation. Pertinences interprétatives et surinterprétations en sciences sociales, Enquête, n³, pp. 61-89.

Latouche, S., Laurent P.-J., Singleton, M., Servais, O., (eds), 2004. Les raisons de la ruse, Paris, La découverte / MAUSS.

Latour, B. 1995. La science en action, Paris, Gallimard.

Laurent, P.-J. 1995. Les pouvoirs politiques locaux et la décentralisation au Burkina Faso, Louvain-laNeuve / Paris, Cahiers du CIDEP n²6, Académia-L'Harmattan.

-. 1998. Une association de développement en pays mossi. Le don comme ruse, Paris, Karthala. 
-. déc. 2000. « Entre ville et campagne : le big man local ou la "gestion coup d'Etat" de l'espace public », Politique africaine, $n^{\circ} 80$, pp. 169-181.

-. 2000. «Sémantique populaire du détournement dans les associations de développement, en pays Mossi (Burkina Faso) », in Blundo G. (dir.), Monnayer les pouvoirs. Espaces, mécanismes et représentations de la corruption, Génève - Paris, Cahiers de IUED - PUF, n 9 , pp. 221-248.

-. 2003. Les pentecôtistes du Burkina. Mariage, pouvoir et guérison, Paris, coédition IRD-Karthala (col.

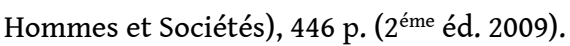

-. 2006. «L'implication de l'anthropologue dans le champ politique : l'exemple de la Commission Nationale de la Décentralisation du Burkina Faso ", Recherches sociologiques et anthropologiques, vol. $37, \mathrm{n}^{\circ} 1$, pp. $105-120$.

-. 2008. «Éléments pour une socio-anthropologie de la défiance. L'inhumain et l'humain, esquisse d'une comparaison à partir de la société mossi du Burkina Faso », in Furtos, J. (dir.), Les cliniques de la précarité, Paris, Plon.

-. 2010. Beautés imaginaires. Anthropologie du corps et de la parenté, Louvain-la-Neuve, Academia, Coll. Anthropologie prospective.

-. 2011. «Système de mariages et terminologies de la parenté chez les Mossi. Contribution à l'approche de la terminologie omaha », Paris, soumis pour publication : L'Homme (novembre 2011).

Lévinas, E. 2006. Totalité et infini. Essai sur l'extériorité, La Haye, Nijhoff, Livre de Poche (éd. or. 1971).

Lévi-Strauss, C. 2008. Euvres. Le totémisme aujourd'hui, Paris, Gallimard (éd. or. PUF, 1962).

Liendo, R. 2009. Participación popular y movimiento campesino aymara, thèse de doctorat, Louvain-laNeuve, UCL. ESPO/SPED - Département des sciences de la population et du développement.

Mahmood, S. 2009. Politique de la piété. Le féminisme à l'épreuve du renouveau islamique, Paris, La découverte.

Mazzocchetti, J. 2007Aspects de la jeunesse universitaire de Ouagadougou au Burkina Faso. Lorsque dire c'est faire : une ethnologie des imaginaires, Louvain-la-Neuve, thèse de doctorat.

Médard, J.-F. 1992. «Le big man en Afrique. Du politicien entrepreneur », L'Année sociologique, $\mathrm{n}^{\circ} 42$, pp. 167-192.

Nke Ndih, J. 2002. «Comment peut-on être écologiste en Afrique? », Le Monde Diplomatique, décembre 2002 .

-. 2006. « Du campement aux enjeux planétaires: les populations Pygmées à la croisée des chemins ", in Abega, S-C. et Bigombe Logo, P. (eds), La marginalisation des Pygmées d'Afrique centrale, Paris, Maisonneuve et Larose, pp. 257-270.

Olivier de Sardan, J.-P. 1991. « L'anthropologie du changement social et du développement comme ambition théorique ?», Bulletin de l'APAD, n¹, pp. 7-11.

-. 2008, La rigueur du qualitatif. Les contraintes empiriques de l'interprétation socio-anthropologiques, Louvain-la-Neuve, Académia.

-. 2001. «Les trois approches en anthropologie du développement », Revue Tiers Monde, t. XLII, n¹68, oct.-déc., pp. 729-754.

-. 1996. « La violence faite aux données ", Enquête, $\mathrm{n}^{\circ} 3$, pp. 31-60. 
Ricoeur, P. 1997. L'idéologie et l'utopie, Paris, Seuil.

-. 1969. Le conflit des interprétations, Paris, Seuil.

-. 2004. Parcours de la reconnaissance. Trois études, Paris, Stock, Morin, E., La méthode 6.

Ethique, Paris, Seuil, pp. 121-139.

-. 1990. Soi-même comme un autre, Paris, Seuil, 423 p.

Sahlins, M. 1963. « Poor man, rich man, big man, chief: Political types », in Melanesia and Polynesia, Comparative Studies in Society and History, 5, pp. 285-303.

Said W., E. 2000. Culture et impérialisme, Paris, Fayardp.

Saillant, F. (ed.) 2009. Réinventer l'anthropologie ? Les sciences de la culture à l'épreuve des globalisations, Montréal, Liber.

Saillant, F., Kilani M., Graezer Bideau F. (eds), 2011. Manifeste de Lausanne. Pour une anthropologie non hégémonique, Montréal, Liber.

Singleton, M. 1991. «Projet et projets ", Cahiers du CIDEP, $n^{\circ} 7$.

-. 1991. «Souci d'Autrui : entre Charité cannibale et l'altruisme anthropophage », Cahiers du CIDEP, $\mathrm{n}^{\circ} 10,91 \mathrm{p}$.

-. 1992. «Le Projet APAD et le Projet du peuple », Bulletin de l'APAD, n², pp. 26-30

-. 1993. «Vive la différence ! ", Cahiers du CIDEP, n, n¹6.

-. 1998. Amateurs de chiens à Dakar. Plaidoyer pour un interprétariat anthropologique, Louvain-laNeuve/Paris, Académia/L'Harmattan.

-. 2001. «De l'épaississement empirique à l'interpellation interprétative en passant par l'ampliation analogique : une méthode pour l'anthropologie prospective », Recherches Sociologiques, 1, pp. 15-40.

-. 2004. Critique de l'ethnocentrisme. Du missionnaire anthropologue à l'anthropologue postdéveloppementiste, Paris, Parangon.

-. 2009. «Saint George et le dragon? Quelle altérité pour quel avenir disciplinaire ", in Saillant, F. (sous la dir. de) Réinventer l'anthropologie? Les sciences de la culture à l'épreuve des globalisations, Montréal, Liber, pp. 23-44.

Tonda, J. 2002. La guérison divine en Afrique centrale (Congo, Gabon), Paris, Karthala.

Veyne, P. 1971. Comment on écrit l'histoire. Essai d'épistémologie, Paris, Seuil.

\section{NOTES}

1. Pour une approche de cette question en anthropologie voir Laurent, P.-J., «L'implication de l'anthropologue dans le champ politique: l'exemple de la Commission Nationale de la Décentralisation du Burkina Faso ", Recherches sociologiques et anthropologiques, vol. 37, n¹, 2006, pp. 105-120.

2. Pour une critique de l'engagement politique en science, voir par exemple: Mahmood, S., Politique de la piété. Le féminisme à l'épreuve du renouveau islamique, Paris, La découverte, 2009.

3. Voir par exemple: Said, W., E., Culture et impérialisme, Paris, Fayard 2000, 555 p. ; voir aussi : Bhabha, H., K., Paris, Payot, Les lieux de la culture : Une théorie postcoloniale 2007. 
4. Bensa, A., La fin de l'exotisme. Essais d'anthropologie critique, Toulouse, Anacharis, 2006, 372 p.; Bensa, A., De la relation ethnographique. A la recherche de la juste distance, Enquête, n¹, 1995, pp. 131-140.

5. Geertz, G., Bali. Interprétation d'une culture, Paris, Gallimard, (notamment, Jeu d'enfer. Notes sur le combat de coqs balinais), 1983, pp. 166 - 215.

6. J'y ai ensuite effectué plus de cinquante missions brèves entre 1991 et 2010

7. Cf. : Laurent, P.-J., Une association de développement en pays mossi. Le don comme ruse, Paris, Karthala, (collec. Hommes et Sociétés), 1998.

8. Selon cette belle formule dont Mike Singleton a le secret.

9. Pour une analyse instructive de cette situation, à la faveur de la relecture de l'interprétation de la faune fossilisée des Schistes de Burgess, voir: Gould, S., J., La vie est belle. Les surprises de l'évolution, Paris, Seuil, 1989.

10. Cf. : Blundo, G., Olivier de Sardan, J.-P., Pratiques de la description, Paris, EHESS, Enquête n³, 2003, 223 p.; Lahire, B., Risquer l'interprétation. Pertinences interprétatives et surinterprétations en sciences sociales, Enquête, n³, 1996, pp. 61-89.; Olivier de Sardan,J.-P., La violence faite aux données, Enquête, n³, 1996, pp. 31-60.

11. Voir notamment : Godelier, M., Métamorphoses de la parenté, Paris, Fayard, 2004, 678 p.; pour un débat critique de cette question dans le champ particulier de l'anthropologie fondamentale : Laurent, P.-J., Beautés imaginaires. Anthropologie du corps et de la parenté, Louvain-la-Neuve, Académia, Coll. Anthropologie prospective, 2010.

12. Voir notamment: Bierschenk, $T$., «Les projets et les politiques de développement sont-ils des préoccupations légitimes de l'anthropologie?», Bulletin de l’APAD, 1991, n¹, pp. 12-14.; Chauveau, J.-P., «Le modèle participatif» de développement rural est-il «alternatif»? (Eléments pour une anthropologie de la culture des "développeurs »), Bulletin de l'APAD, 1992, $\mathrm{n}^{\circ} 3$, pp. 20-30.; Olivier de Sardan, J.-P., «L'anthropologie du changement social et du développement comme ambition théorique? ", Bulletin de l’APAD, 1991, n¹, pp. 7-11. ; Singleton, M., « Le Projet APAD et le Projet du peuple », Bulletin de l'APAD, n², 1992, pp. 26-30.

13. Mazzocchetti, J., Aspects de la jeunesse universitaire de Ouagadougou au Burkina Faso. Lorsque dire c'est faire : une ethnologie des imaginaires, Louvain-la-Neuve, thèse de doctorat, 2007, 343 p. ; par ailleurs, Jackie Assayag a montré que la globalisation n'est ni bonne ni mauvaise en soit, mais qu'elle s'appréhende par la nature et le fonctionnement des rapports sociaux à l'échelle locale. Elle démontre ainsi la nécessité d'articuler les études locales à celles qui les relient au global (Assayag, J., La mondialisation vue d'ailleurs. L'Inde désorientée, Paris, Seuil, pp. 177$222,2005)$

14. Singleton, M., Critique de l'ethnocentrisme. Du missionnaire anthropologue à l'anthropologue postdéveloppementiste, Paris, Parangon, 2004.

15. Singleton, M., Amateurs de chiens à Dakar. Plaidoyer pour un interprétariat anthropologique, Louvain-la-Neuve/Paris, Académia/L'Harmattan, 1998 ; Singleton, M., Critique de l'ethnocentrisme.... Op. cit.. ; voir aussi Singleton, M., «Projet et projets », Cahiers $d u$ CIDEP, $n^{\circ} 7$, 1991 ; Singleton, M., «Souci d'Autrui : entre Charité cannibale et l'altruisme anthropophage », Cahiers du CIDEP, n¹0, 1991 ; Singleton, M., "Vive la différence ! ", Cahiers du CIDEP, n7, 1993, $\mathrm{n}^{\circ} 16$; Singleton, M. De l'épaississement empirique à l'interpellation interprétative en passant par l'ampliation analogique : une méthode pour l'anthropologie prospective, Recherches Sociologiques, 1, 2001, pp. 15-40 ; Singleton, M., Saint George et le dragon? Quelle altérité pour quel avenir disciplinaire, in Saillant, F. (sous la dir. de) Réinventer l'anthropologie? Les sciences de la culture à l'épreuve des globalisations, Montréal, Liber, 2009, pp. 23-44.

16. Par exemple : Saillant, F. (sous la dir. de) Réinventer l'anthropologie ? Les sciences de la culture à l'épreuve des globalisations, Montréal, Liber, 2009.

17. Cf. notamment Agier, M., La sagesse de l'ethnologue, Paris, L'œil neuf, 2004. 
18. A ce propos : Lévinas, E., Totalité et infini. Essai sur l'extériorité, La Haye, Nijhoff, Livre de Poche, 2006 (éd. or. 1971).

19. Cf. Ricoeur, P., Soi-même comme un autre, Paris, Seuil, 1990.

20. A ce propos voir également : Descola, P., Par delà nature et culture, Paris, Gallimard, 2005, 623 p. Pour une critique assez radical similaire à celle développée à Mike Singleton : Butler, J., Trouble dans le genre. Le féminisme et la subversion de l'identité, Paris, La découverte, 2005.

21. Olivier de Sardan, J.-P., La rigueur du qualitatif. Les contraintes empiriques de l'interprétation socioanthropologiques, Louvain-la-Neuve, Académia, 2008.

22. Pour un essai récent consacré à la question : Saillant, F., Kilani, M., Graezer Bideau, F. (sous la dir. de), Manifeste de Lausanne. Pour une anthropologie non hégémonique, Montréal, Liber, 2011.

23. Kilani, M., L'invention de l'autre. Essais sur le discours anthropologique, Lausanne, Payot, 1994 ; voir aussi : Nke Ndih, J., Comment peut-on être écologiste en Afrique?, Le Monde Diplomatique, décembre 2002 ; Nke Ndih, J., Du campement aux enjeux planétaires: les populations Pygmées à la croisée des chemins, in Abega, S-C. et Bigombe Logo, P. La marginalisation des Pygmées d'Afrique centrale, Paris, Maisonneuve et Larose, 2006, pp. 257-270. ; Liendo, R., Participación popular y movimiento campesino aymara, thèse de doctorat, Louvain-la-Neuve, UCL. ESPO/SPED Département des sciences de la population et du développement, 2009.

24. Pour un débat de cette notion : Laurent, P-J., Les pentecôtistes du Burkina. Mariage, pouvoir et guérison, Paris, coédition IRD-Karthala (col. Hommes et Sociétés), 2003 (Seconde édition, ave nouvelle introduction 2009).

25. . Voir P.-J. Laurent, Une association de développement en pays mossi. Le Don comme ruse, Paris, Karthala, 1998.

26. Pour une explique détaillée de ces deux cas: Laurent, P.-J., "Sémantique populaire du détournement dans les associations de développement, en pays Mossi (Burkina Faso), in Monnayer les pouvoirs. Espaces, mécanismes et représentations de la corruption. Sous la dir. de Blundo, G., Génève - Paris, Cahiers de IUED - PUF, $\mathrm{n}^{\circ} 9,2000$, pp. 221-248.

27. Cet état de confiance au sein des familles, entres affins et consanguins, est précaire et toujours à reconstruire face aux forces centrifuges dont celles d'accusations de sorcelleries. Pour une description détaillée de ces processus : Laurent, P.-J., Système de mariages et terminologies de la parenté chez les Mossi. Contribution à l'approche de la terminologie omaha, Paris, soumis pour publication : L'Homme (novembre 2011), $28 \mathrm{p}$.

28. Wuum taaba : litt. comprendre ses camarades, renvoie à ce que j'ai désigné par l'idéologie de «l'entente ». Il s'agit en quelque sorte d'un agrément, soit de consentir à quelque chose, dans le sens d'une approbation émanant d'une autorité.

29. Laurent, P.-J., "Éléments pour une socio-anthropologie de la défiance. L'inhumain et l'humain, esquisse d'une comparaison à partir de la société mossi du Burkina Faso ", in Furtos, J. (dir.), Les cliniques de la précarité, Paris, Plon, 2008.

30. Pour des détails: Laurent, P.-J., Les pouvoirs politiques locaux et la décentralisation au Burkina Faso, Louvain-la-Neuve / Paris, Cahiers du CIDEP n²6, Académia-L'Harmattan, 1995.

31. Voir à ce propos l'idée du «Souverain moderne" de Joseph Tonda, in Tonda, J., La guérison divine en Afrique centrale (Congo, Gabon), Paris, Karthala, 2002.

32. Laurent, P.-J., Entre ville et campagne : le big man local ou la "gestion coup d'Etat " de l'espace public, Politique africaine, n80, déc. 2000, pp. 169-181.) J'utilise le terme de big man dans le sens ethnographique de self-made man: c'est un leader qui s'impose à un groupe par son charisme, sa propre force ou habileté et non par héritage de sa fonction d'un ascendant. Voir les sociétés à big men de Mélanésie décrites entre autres (voir aussi M. Godelier) par M. Sahlins, "Poor man, rich man, big man, chief: Political types ", in Melanesia and Polynesia, Comparative Studies in Society and History, 5, 1963, pp. 285-303. Jean-François Médard avait déjà proposé ce rapprochement entre le big man anthropologique et le big man de l'Afrique contemporaine : J.- 
F. Médard, « Le big man en Afrique. Du politicien entrepreneur », L’Année sociologique, n 42, 1992, pp. 167-192.

33. Laurent, P.-J., Entre ville et campagne..., op. cit.

34. Autour de la culture hybride, ou encore de l'idée de "l'état inachevé de la société de contrat", voir par exemple: Da Matta, R., Carnavals, bandits et héros : ambiguités de la société brésilienne, Paris, Seuil, 1983 ; Garcia-Canclini, N., Culturas hibridas. Estrategias para entrar y salir de la modernidad, Editorial Sudamericana, 2da edición, Buenos Aires, 1995.

35. Sur cette notion: Olivier de Sardan, J.-P., Les trois approches en anthropologie du développement, Revue Tiers Monde, t. XLII, n¹68, oct.-déc., 2001, pp. 729-754.

36. Laurent, P.-J., "Sémantique populaire du détournement dans les associations de développement, en pays Mossi (Burkina Faso)», op. cit.

37. Sur la formulation de cette opposition: Bourdieu, P., Comprendre, in Bourdieu, P. (sous la dir. de) La misère du monde, Paris, Seuil, 1993, pp. 903-939.

38. Sur cette vaste thématique : Ricoeur, P., L'idéologie et l'utopie, Paris, Seuil, 413 p. ; Latour, B., La science en action, Paris, Gallimard, 1995.

39. A propos de l'éthique de la connaissance : Ricoeur, P., Parcours de la reconnaissance. Trois études, Paris, Stock, 2004, 386 p. Voir aussi Morin, E., La méthode 6. Ethique, Paris, Seuil, pp. 121-139.

\section{RÉSUMÉS}

Cet article présente des jalons pour une anthropologie résolument préoccupée par des enjeux épistémologiques et éthiques renouvelés au contact des terrains du XXIe siècle lesquels posent de lourds défis à la discipline dont ceux portés par la critique postcoloniale et la nécessité de s'émanciper du terrain conçu comme une entité close afin de sortir définitivement de l'exotisme.Impliqué depuis la création de l'APAD dans des recherches sur le développement et le changement social, j'avais, avant ma carrière universitaire, piloté des projets de développement et accepté des missions d'expertise comme agronome et anthropologue.

This article presents the bases for an anthropology resolutely concerned with renewed epistemological and ethical issues posed by the contact with "the field" in the 21st century. These challenges to the discipline include those of the postcolonial critique and of the need to liberate oneself from the conception of a bounded field in order to put a definite end to exoticism. This reflexive article is based both on my involvement, since the creation of APAD, in research on development and social change, and on a previous career in development projects and expert assignments as an agronomist and anthropologist. 